\title{
Clinical Characteristics and Treatment Outcomes of Pediatric Patients with Non-Hodgkin Lymphoma in East Asia
}

\section{Jin Kyung Suh, MD, PhD' \\ Yi-Jin Gao, MD² \\ Jing-Yan Tang, MD2 \\ Shiann-Tarng Jou, $\mathrm{MD}^{3}$ \\ Dong-Tsamn Lin, $\mathrm{MD}^{3}$ \\ Yoshiyuki Takahashi, MD ${ }^{4}$ \\ Seiji Kojima, MD ${ }^{4}$ \\ Ling Jin, $\mathrm{MD}^{5}$ \\ Yonghong Zhang, $\mathrm{MD}^{5}$ \\ Jong Jin Seo, MD, PhD'}

${ }^{1}$ Division of Pediatric Hematology/Oncology, Department of Pediatrics, Asan Medical Center Children's Hospital, University of Ulsan College of Medicine, Seoul, Korea, ${ }^{2}$ Department of Hematology/Oncology, Shanghai Children's Medical Center, Shanghai, China, ${ }^{3}$ Department of Pediatrics Hematology/Oncology, National Taiwan University Hospital, Taipei, Taiwan,

${ }^{4}$ Department of Pediatrics, Nagoya University Graduate School of Medicine, Nagoya, Japan,

${ }^{5}$ Hematology Oncology Center,

Beijing Children's Hospital,

Capital Medical University, Beijing, China

Correspondence: Jong Jin Seo, MD, PhD Division of Pediatric Hematology/Oncology, Department of Pediatrics, Asan Medical Center Children's Hospital, University of Ulsan College of Medicine, 88 Olympic-ro 43-gil, Songpa-gu, Seoul 05505, Korea

Tel: 82-2-3010-3383

Fax: 82-2-473-3725

E-mail: jjseo@amc.seoul.kr

Co-correspondence: Yonghong Zhang, MD Hematology Oncology Center, Beijing Children's Hospital, Capital Medical University,

56 Nan Lishi Road, Beijing 100045, China

Tel: 86-10-59617623

Fax: 86-13701251969

E-mail: yhzhang58@hotmail.com

Received April 22, 2019

Accepted July 26, 2019

Published Online July 29, 2019

*Jin Kyung Suh and Yi-Jin Gao contributed equally to this work.

\section{Purpose}

The presentations and geographic incidence of pediatric non-Hodgkin lymphoma (NHL) differ from those of adults. This study delineated the characteristics and outcomes of pediatric $\mathrm{NHL}$ in East Asia.

\section{Materials and Methods}

Medical records of 749 pediatric patients with NHL treated at participating institutions in mainland China, Japan, Korea, and Taiwan from January 2008 to December 2013 were reviewed. Demographic and clinical features, survival outcomes, and putative prognostic factors were analyzed.

\section{Results}

Five hundred thirty patients (71\%) were male. The most common pathologic subtypes were Burkitt lymphoma (BL) (36\%). Six hundred seven patients (81\%) had advanced diseases at diagnosis. The 5-year overall survival and event-free survival (EFS) rates were $89 \%$ and $84 \%$. The 5-year EFS rates of BL, lymphoblastic lymphoma, and diffuse large B-cell lymphoma were $88 \%, 88 \%$, and $89 \%$, and those of anaplastic large cell lymphoma (ALCL) and peripheral T-cell lymphoma (PTCL) were $71 \%$ and $56 \%(p<0.001)$. Central nervous system involvement, high lactate dehydrogenase level (> $250 \mathrm{lU} / \mathrm{mL})$, and advanced disease at diagnosis ( $\geq$ stage III) were associated with poor outcomes $(p<0.05)$. ALCL and PTCL relapsed more frequently than other pathologic subtypes $(p<0.001)$.

\section{Conclusion}

In East Asia, PTCL was more frequent than in Western countries, and bone marrow involvement did not affect treatment outcome. This international study should motivate future collaborative study on NHL in East Asia.

\section{Key words}

Lymphoma, Child, Treatment outcome, Retrospective studies 


\section{Introduction}

Non-Hodgkin lymphoma (NHL) is a heterogeneous group of lymphoid malignancies and is the fourth most common malignancy in children and adolescents. Pediatric NHLs are known to have different distributions of pathologic subtypes and clinical features compared to adult NHLs. Most pediatric patients with NHL present diffuse high-grade lymphomas with frequent extranodal involvement $[1,2]$. Treatment outcomes of pediatric NHL have been improved with effective treatment strategies according to different pathologic subtypes, and the current overall survival (OS) rate exceeds $80 \%$ [3]. However, most of these findings on pediatric NHLs are derived from studies in Western countries. Multicenter investigations on Asian patients with pediatric NHL are lacking except for a few nationwide studies in Japan, China, and South Korea [4-6]. This might be attributed to the heterogeneity and the low incidence of pediatric NHL, which prevents large-scale clinical studies. Therefore, in this study we analyzed the demographic and clinical features of a large number of pediatric patients with NHL in Asian countries and compared them with those of previous reports on patients in Western countries to provide a basis for further clinical investigation.

\section{Materials and Methods}

The medical records of 769 pediatric patients with NHL, who were diagnosed and treated in four participating institutions of Beijing, Shanghai, Nagoya, and Seoul, and in centers in Taiwan between January 2008 and December 2013, were reviewed retrospectively. Participating centers were the Asan Medical Center Children's Hospital (AMCCH) in Korea, Nagoya University Hospital (NUH) in Japan, Beijing Children's Hospital (BCH) and Shanghai Children's Medical Center in China, and Taiwan Pediatric Oncology Group centers. Demographic, clinical features, and survival outcomes were investigated.

NHL was diagnosed and classified according to the World Health Organization Classification of Hematological Malignancies [7]. Central review of the diagnosis was not performed. The St. Jude staging system was used for staging at diagnosis [8]. Staging analysis included physical examination, peripheral blood smear, and bone marrow (BM) aspiration and biopsy, cerebrospinal fluid (CSF) analysis, computed tomography (CT) and / or magnetic resonance imaging (MRI), fluorodeoxyglucose positron emission tomography and serum lactate dehydrogenase (LDH) concentration. Ini- tial central nervous system (CNS) disease was diagnosed if one of the following was present: lymphoma cells in the CSF ( $>5$ cells / $\mu \mathrm{L}$ CSF in patients with lymphoblastic lymphoma [LL]), cerebral infiltrates on cranial CT or MRI, or cranial nerve palsy not caused by an extradural mass. Patients with blasts of $\geq 25 \%$ in BM were diagnosed with acute lymphoblastic lymphoma and were not included in this study. However, exclusively in patients with Burkitt lymphoma (BL), those with BM involvement were included as stage IV regardless of the percentage of blasts in the BM.

Patients were treated with multi-agent chemotherapy reg-

Table 1. Patients' characteristics

\begin{tabular}{|c|c|}
\hline Characteristic & No. $(\%)(\mathrm{n}=749)$ \\
\hline Age at diagnosis, median (range, yr) & $7.5(0.6-18.6)$ \\
\hline \multicolumn{2}{|l|}{ Sex } \\
\hline Male & $530(71.0)$ \\
\hline Female & $219(29.0)$ \\
\hline \multicolumn{2}{|l|}{ Pathologic subtype } \\
\hline$B L^{a)}$ & $268(35.7)$ \\
\hline LL & $224(29.9)$ \\
\hline DLBCL & 97 (12.9) \\
\hline ALCL & $107(14.3)$ \\
\hline $\mathrm{PTCL}^{\mathrm{b})}$ & $35(4.6)$ \\
\hline Others ${ }^{c}$ & $18(2.4)$ \\
\hline $\begin{array}{l}\text { LDH level at diagnosis, } \\
\text { median (range, IU/mL) }\end{array}$ & $500(100-9,587)$ \\
\hline \multicolumn{2}{|l|}{$\begin{array}{l}\text { BM involvement } \\
\text { (of the evaluable } 748 \text { patients) }\end{array}$} \\
\hline Yes & $217(29.0)$ \\
\hline No & $531(70.8)$ \\
\hline \multicolumn{2}{|l|}{ CNS involvement } \\
\hline Yes & $63(8.4)$ \\
\hline No & $686(91.6)$ \\
\hline \multicolumn{2}{|l|}{ St. Jude stage } \\
\hline I & $28(3.7)$ \\
\hline II & $113(15.1)$ \\
\hline III & $368(49.1)$ \\
\hline IV & $240(32.0)$ \\
\hline
\end{tabular}

BL, Burkitt lymphoma; LL, lymphoblastic lymphoma; DLBCL, diffuse large B-cell lymphoma; ALCL, anaplastic large cell lymphoma; PTCL, peripheral T-cell lymphoma; $\mathrm{LDH}$, lactate dehydrogenase; $\mathrm{BM}$, bone marrow; CNS,

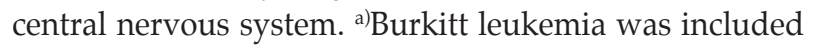
in BL, b)PTCL-not otherwise specified, natural killer/T-cell lymphoma and subcutaneous panniculitis T-cell lymphoma were included in PTCL, c)Others: mucosa-associated lymphoid tissue lymphoma, other unspecified B-NHL, follicular lymphoma, hydrovascciniforme-like lymphoma, plasmablastic lymphoma. 

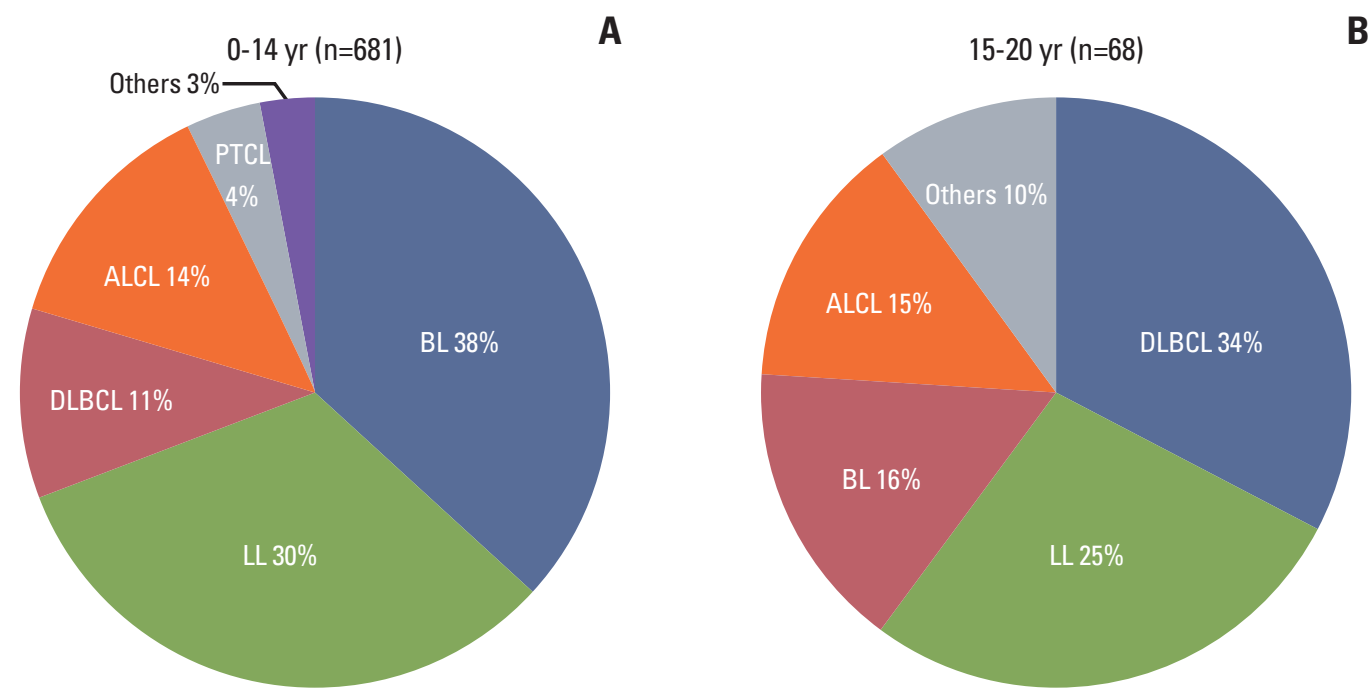

Fig. 1. Pathologic subtype distribution according to age. BL was the most common subtype in patients under 14 years old (A), while DLBCL was in patients aged 15-20 years old (B). BL, Burkitt lymphoma; LL, lymphoblastic lymphoma; DLBCL, diffuse large B-cell lymphoma; ALCL, anaplastic large cell lymphoma; PTCL, peripheral T-cell lymphoma.

imens according to participating institutions. Most of the regimens adopted the backbone of NHL-Berlin-Frankfurt-Munster (BFM) or Children's Oncology Group (COG) protocols, which were stratified to the three main subgroups of LL, mature B-cell lymphoma, and anaplastic large cell lymphoma (ALCL).

Analysis of the probability of OS and event-free survival (EFS) was performed using the Kaplan-Meier method with differences compared by the log-rank test. The EFS was calculated from the date of diagnosis to the first event (first relapse, progression, secondary malignancy, or death from any cause) or the date of last follow-up. Cumulative incidence (CI) functions for competing events were constructed by the method of Kalbfleisch and Prentice and were compared with Gray's test. CIs of relapse were estimated considering death without relapse and secondary malignancies as competing events. Differences in the distributions of different parameters were examined using the chi-square or Fisher exact test. Statistical analysis was conducted using SPSS software ver. 23.0 (IBM Corp., Armonk, NY) and R ver. 3.1.1 (R Foundation for Statistical Computing, Vienna, Austria). p-values $<0.05$ were considered statistically significant.

\section{Ethical statement}

This study was approved by the Institutional Review Board (IRB) of the Asan Medical Center Children's Hospital (No. 2020-0284). Due to retrospective nature of this study, informed consent was waived by IRB. This study was performed in accordance with the recommendations of the Dec- laration of Helsinki for biomedical research involving human subjects.

\section{Results}

A total of 769 patients were enrolled in this study. Of them, 20 cases were excluded due to inadequate data. Patient characteristics are summarized in Table 1. Patients showed male predominance (male vs. female, $71 \%$ vs. $29 \%$ ). The most common pathologic subtype was BL (36\%) followed by LL (30\%), ALCL (14\%), and diffuse large B-cell lymphoma (DLBCL) $(13 \%)$. Other rare lymphomas $(2 \%)$ included seven other unspecified B-NHLs, five follicular lymphomas, three mucosa-associated lymphoid tissue lymphomas, two hydrovacciniforme-like lymphomas, and one plasmablastic lymphoma. There were slight differences in the pathologic subtype distributions according to the participating institutions and age. LL was more common in BCH, BL was more frequently observed in NUH, peripheral T-cell lymphoma (PTCL) was more often observed in AMCCH, and DLBCL was more common in Taiwan. In the analysis according to age, BL was predominant among patients under 14 years old while DLBCL was predominant among those aged 15-20 years (Fig. 1). Most patients (81\%) had advanced disease ( $\geq$ stage III) at diagnosis. CNS involvement and BM involvement were observed in $63(8 \%)$ and $217(29 \%)$ patients, respectively. Anaplastic lymphoma kinase (ALK) status was avail- 
Table 2. BM, CNS involvement and disease extent at diagnosis according to pathologic subtype

\begin{tabular}{|c|c|c|c|c|c|c|c|c|}
\hline & $\begin{array}{c}\text { BL } \\
(n=268)\end{array}$ & $\begin{array}{c}\mathrm{LL}^{\mathrm{a})} \\
(\mathrm{n}=\mathbf{2 2 4})\end{array}$ & $\begin{array}{c}\text { ALCL } \\
(\mathbf{n = 1 0 7 )}\end{array}$ & $\begin{array}{c}\text { DLBCL } \\
(n=97)\end{array}$ & $\begin{array}{l}\text { PTCL } \\
(n=35)\end{array}$ & $\begin{array}{l}\text { Others } \\
(n=18)\end{array}$ & $\begin{array}{c}\text { Total } \\
(n=749)\end{array}$ & p-value \\
\hline \multicolumn{9}{|c|}{ BM involvement } \\
\hline Yes & $81(30.2)$ & $108(48.2)$ & $12(11.2)$ & $9(9.3)$ & $2(5.7)$ & $5(27.7)$ & $217(28.9)$ & $<0.001$ \\
\hline No & $187(69.8)$ & $115(51.3)$ & $95(88.8)$ & $88(90.7)$ & $33(94.2)$ & $13(72.2)$ & $531(70.9)$ & \\
\hline \multicolumn{9}{|c|}{ CNS involvement } \\
\hline Yes & 32 (11.9) & $17(7.6)$ & $5(4.7)$ & $8(8.2)$ & $1(2.8)$ & 0 & $63(8.4)$ & 0.087 \\
\hline No & $236(88.1)$ & $207(92.4)$ & $102(95.3)$ & 89 (91.8) & $34(97.1)$ & $18(100)$ & $686(91.5)$ & \\
\hline \multicolumn{9}{|c|}{ Advanced disease (stage $\geq 3$ ) } \\
\hline Yes & $222(82.8)$ & $209(93.3)$ & $85(79.4)$ & $66(68.0)$ & $18(51.4)$ & $10(55.5)$ & $610(81.4)$ & $<0.001$ \\
\hline No & $46(17.2)$ & $15(6.7)$ & $22(20.6)$ & $31(32.0)$ & $17(48.5)$ & $8(44.4)$ & $139(18.5)$ & \\
\hline
\end{tabular}

Values are presented as number (\%). BM, bone marrow; CNS, central nervous system; BL, Burkitt lymphoma; LL, lymphoblastic lymphoma; ALCL, anaplastic large cell lymphoma; DLBCL, diffuse large B-cell lymphoma; PTCL, peripheral T-cell lymphoma. ${ }^{\text {a) }}$ One patient with LL did not have data of initial BM.
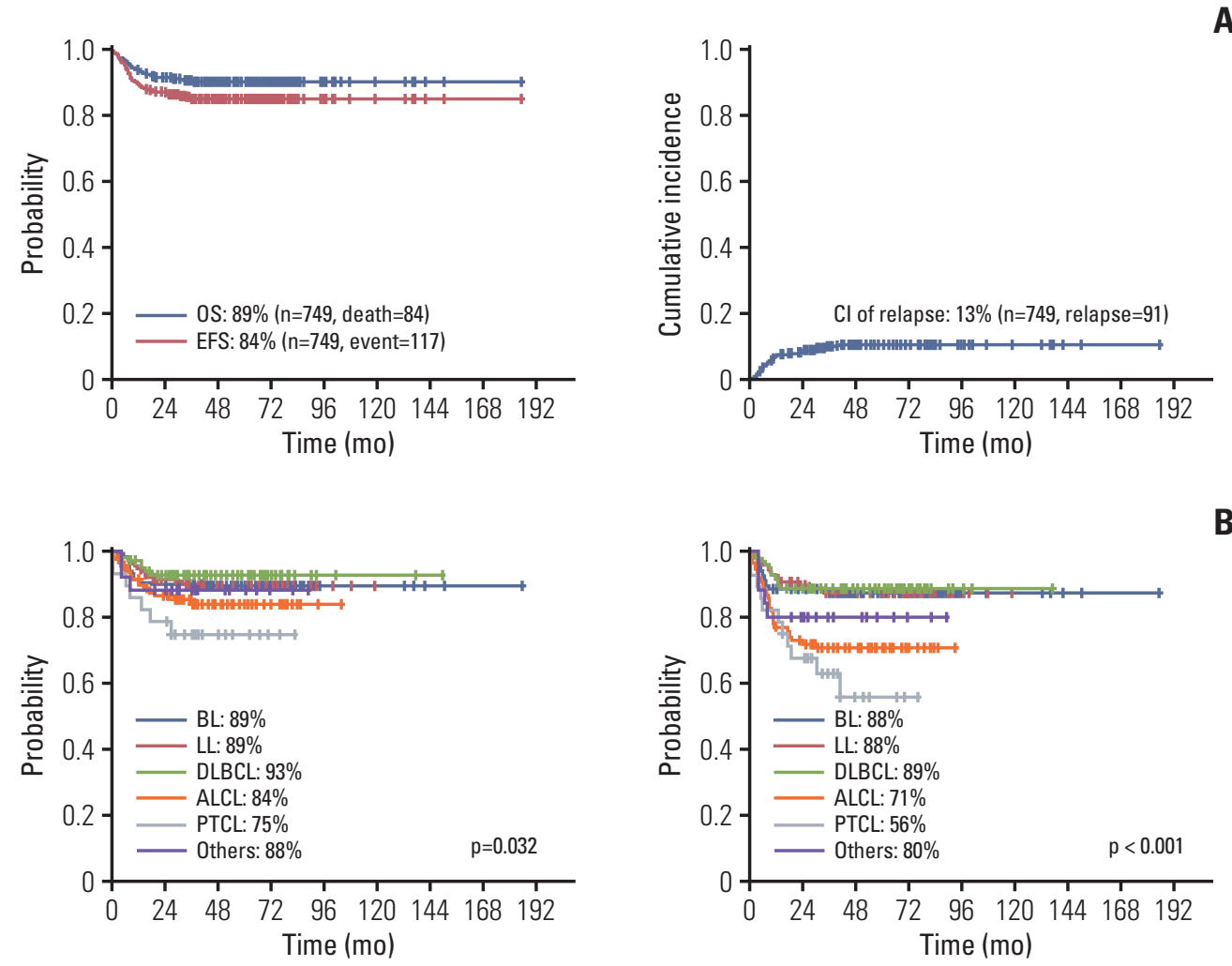

Fig. 2. Treatment outcomes of the 749 pediatric patients with NHL (A) and treatment outcomes according to pathologic subtypes (B). OS, overall survival; EFS, event-free survival; CI, confidence interval; BL, Burkitt lymphoma; LL, lymphoblastic lymphoma; DLBCL, diffuse large B-cell lymphoma; ALCL, anaplastic large cell lymphoma; PTCL, peripheral T-cell lymphoma. 
Table 3. Univariate analysis of putative prognostic factors

\begin{tabular}{|c|c|c|c|c|c|}
\hline Factor & No. & 5-Year OS (\%) & p-value & 5-Year EFS (\%) & p-value \\
\hline \multicolumn{6}{|l|}{ Sex } \\
\hline Male & 530 & 89 & 0.678 & 84 & 0.651 \\
\hline Female & 219 & 88 & & 83 & \\
\hline \multicolumn{6}{|c|}{ Age at diagnosis (yr) } \\
\hline$\leq 14$ & 681 & 88 & 0.383 & 84 & 0.289 \\
\hline$>14$ & 68 & 92 & & 80 & \\
\hline \multicolumn{6}{|c|}{ Pathologic subtype } \\
\hline BL & 268 & 89 & 0.032 & 88 & $<0.001$ \\
\hline LL & 224 & 89 & & 88 & \\
\hline DLBCL & 97 & 93 & & 89 & \\
\hline ALCL & 107 & 84 & & 71 & \\
\hline PTCL & 35 & 75 & & 56 & \\
\hline Others & 18 & 88 & & 80 & \\
\hline \multicolumn{6}{|c|}{ BM involvement } \\
\hline Yes & 217 & 86 & 0.479 & 81 & 0.482 \\
\hline No & 531 & 89 & & 85 & \\
\hline \multicolumn{6}{|c|}{ CNS involvement } \\
\hline Yes & 63 & 76 & 0.003 & 74 & 0.019 \\
\hline No & 686 & 89 & & 85 & \\
\hline \multicolumn{6}{|c|}{ St. Jude stage } \\
\hline I-II & 141 & 98 & $<0.001$ & 94 & $<0.001$ \\
\hline III-IV & 608 & 86 & & 82 & \\
\hline \multicolumn{6}{|c|}{ Initial LDH (IU/mL) } \\
\hline$\leq 250$ & 170 & 96 & $<0.001$ & 93 & $<0.001$ \\
\hline$>250$ & 579 & 86 & & 81 & \\
\hline
\end{tabular}

OS, overall survival; EFS, event-free survival; BL, Burkitt lymphoma; LL, lymphoblastic lymphoma; DLBCL, diffuse large B-cell lymphoma; ALCL, anaplastic large cell lymphoma; PTCL, peripheral T-cell lymphoma; BM, bone marrow; CNS, central nervous system; LDH, lactate dehydrogenase.

able in 51 patients among 107 patients with ALCL status, and 40 patients $(78 \%)$ of them showed ALK positivity. The differences in the patterns of BM involvement and disease extent at diagnosis, according to pathologic subtype, are shown in Table 2. BM involvement was more frequently observed in LL $(48 \%)$ than in other subtypes, and patients with BL (83\%), LL (93\%), and ALCL (79\%) were more frequently diagnosed with advanced diseases $(p<0.001)$. CNS involvement was more frequently observed in patients with BL $(12 \%)$. However, the differences were not statistically significant $(\mathrm{p}>0.05)$. Median age at diagnosis was 8 years (range, 1 to 19). Median LDH level at diagnosis was 500 IU $/ \mathrm{mL}$ (range, 100 to 17,333). The pathologic subtypes included 268 BLs, 224 LLs, 97 DLBCLs, 107 ALCLs, 35 PTCLs, and 18 other rare lymphomas (others).

Of the 749 patients, $84(11 \%)$ died. During the 50.5 months of median follow-up duration, the 5-year OS, EFS, and CI of relapse were $89 \%, 84 \%$, and $13 \%$, respectively (Fig. 2A). There were no significant differences in OS and EFS between insti- tutions.

Univariate analysis of putative prognostic factors revealed that pathologic subtype, CNS involvement, stage, and initial LDH level were associated with prognosis (Table 3). Patients with PTCL showed significantly poorer prognosis, with 5 -year EFS of $56 \%$, compared with those with other subtypes $(p<0.05)$. CNS involvement at diagnosis was associated with poor survival outcome, while BM involvement was not. Patients with CNS involvement at diagnosis had lower 5-year OS and EFS than patients without CNS involvement $(\mathrm{p}<0.05)$. Patients with early-stage NHL or those with LDH level below $250 \mathrm{IU} / \mathrm{mL}$ at diagnosis had excellent outcomes, with 5-year EFS of over $90 \%$, compared to patients with advanced stage or high level of LDH $(p<0.05)$.

Ninety-one of the 749 patients $(12 \%)$ experienced disease relapse. Median time to relapse from diagnosis was 8 months (range, 0 to 41 months). Along with the factors affecting prognosis, pathologic subtype, CNS involvement and stage were the factors affecting relapse. Patients with ALCL and 
Table 4. Characteristics of relapsed pediatric NHL

\begin{tabular}{|c|c|c|c|c|}
\hline Characteristic & No. $(n=749)$ & Non-relapse group ( $\mathrm{n}=658$ ) & Relapse group $(\mathrm{n}=91)$ & p-value \\
\hline Time from diagnosis to relapse (mo) & & & $7.8(0-41.2)$ & \\
\hline Age at diagnosis $(y r)$ & & $7(1-18)$ & $8.2(0.6-16.4)$ & 0.446 \\
\hline \multicolumn{5}{|l|}{$\operatorname{Sex}(\%)$} \\
\hline Male & 530 & 467 (71.0) & $63(69.2)$ & 0.732 \\
\hline Female & 219 & $191(29.0)$ & $28(30.8)$ & \\
\hline \multicolumn{5}{|l|}{ Pathologic subtype } \\
\hline BL & 268 & $239(36.3)$ & $29(31.9)$ & 0.001 \\
\hline LL & 224 & $203(30.9)$ & $21(23.1)$ & \\
\hline DLBCL & 97 & $90(13.7)$ & $7(7.7)$ & \\
\hline ALCL & 107 & $81(12.3)$ & $26(28.6)$ & \\
\hline PTCL & 35 & $29(4.4)$ & $6(6.6)$ & \\
\hline Others & 18 & $16(2.4)$ & $2(2.1)$ & \\
\hline LDH at diagnosis (IU/mL) & & $476(100-9,587)$ & $610(113-17,333)$ & 0.014 \\
\hline \multicolumn{5}{|l|}{ BM involvement } \\
\hline Yes & 217 & $183(27.8)$ & $35(38.4)$ & 0.098 \\
\hline No & 531 & 475 (72.2) & $58(63.7)$ & \\
\hline \multicolumn{5}{|l|}{ CNS involvement } \\
\hline Yes & 63 & $50(7.5)$ & $13(14.2)$ & 0.031 \\
\hline No & 686 & $608(92.4)$ & $78(85.7)$ & \\
\hline \multicolumn{5}{|l|}{ St. Jude stage } \\
\hline I & 28 & $26(4.0)$ & $2(2.2)$ & 0.005 \\
\hline II & 113 & $108(16.4)$ & $5(5.5)$ & \\
\hline III & 368 & $326(49.5)$ & $41(45.1)$ & \\
\hline IV & 240 & 197 (29.9) & $43(47.2)$ & \\
\hline
\end{tabular}

Values are presented as median (range) or number (\%). NHL, non-Hodgkin lymphoma; BL, Burkitt lymphoma; LL, lymphoblastic lymphoma; DLBCL, diffuse large B-cell lymphoma; ALCL, anaplastic large cell lymphoma; PTCL, peripheral T-cell lymphoma; LDH, lactate dehydrogenase; BM, bone marrow; CNS, central nervous system.

Table 5. Univariate analysis of putative prognostic factors of relapsed pediatric NHL

\begin{tabular}{|c|c|c|c|}
\hline Factor & No. $(n=91)$ & 5-Year OS (\%) & p-value \\
\hline \multicolumn{4}{|c|}{ Median time from diagnosis to relapse (mo) } \\
\hline$\leq 12$ & 68 & 30 & 0.003 \\
\hline$>12$ & 23 & 46 & \\
\hline \multicolumn{4}{|l|}{ Subtype } \\
\hline BL & 29 & 14 & 0.001 \\
\hline LL & 21 & 17 & \\
\hline DLBCL & 7 & 50 & \\
\hline ALCL & 26 & 51 & \\
\hline PTCL & 6 & 75 & \\
\hline Others & 2 & 67 & \\
\hline \multicolumn{4}{|l|}{ Salvage regimen } \\
\hline CTx only & 55 & 36 & $<0.001$ \\
\hline CTx+Auto-HSCT & 10 & 77 & \\
\hline CTx+Allo-HSCT & 10 & 33 & \\
\hline
\end{tabular}

NHL, non-Hodgkin lymphoma; BL, Burkitt lymphoma; LL, lymphoblastic lymphoma; DLBCL, diffuse large B-cell lymphoma; ALCL, anaplastic large cell lymphoma; PTCL, peripheral T-cell lymphoma; CTx, chemotherapy; Auto-HSCT, autologous hematopoietic stem cell transplantation; allo-HSCT, allogeneic hematopoietic stem cell transplantation. 
A

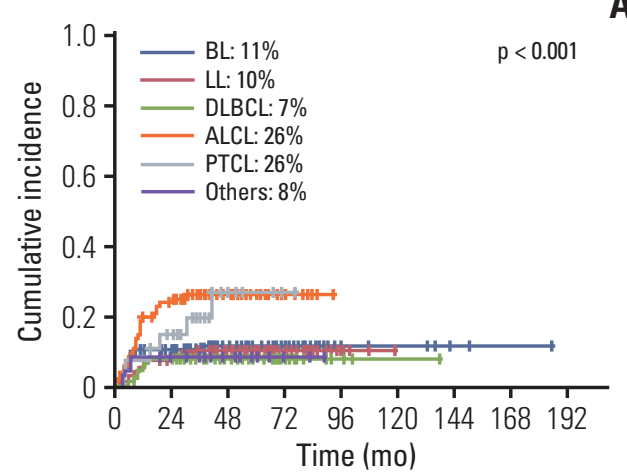

B

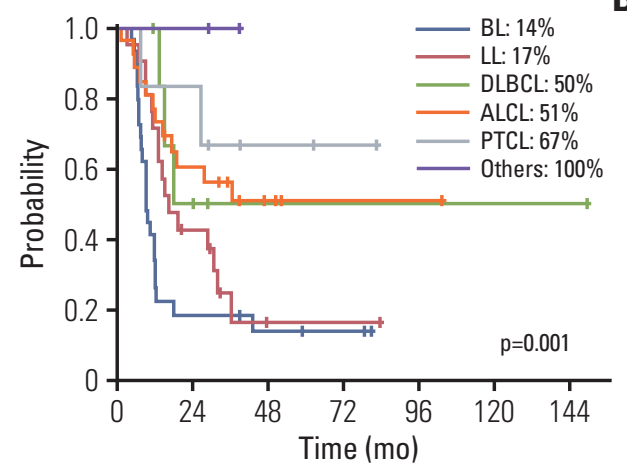

Fig. 3. Treatment outcomes of patients with relapse according to subtype. Cumulative incidence of relapse (A) and overall survival (B) showed that, anaplastic large cell lymphoma (ALCL) and peripheral T-cell lymphoma (PTCL) had a higher incidence of relapse, but they were salvaged successfully compared to Burkitt lymphoma (BL) and lymphoblastic lymphoma (LL). DLBCL, diffuse large B-cell lymphoma.

PTCL experienced relapse more frequently than patients with other subtypes, and patients with CNS involvement and advanced stage also experienced relapses more frequently (Table 4).

Of the 91 patients who experienced relapse, 57 (63\%) died, including the 16 patients who received no treatment after relapse. In the analysis of putative prognostic factors, relapse within 12 months from diagnosis, subtype of BL and LL, and salvage regimen without autologous hematopoietic stem cell transplantation (HSCT) were associated with poor outcomes in patients with relapse (Table 5). ALCL and PTCL showed a higher incidence of relapse (CI of relapse in ALCL and PTCL, 26\%; CI of relapse in BL, LL, and DLBCL: $11 \%, 10 \%$, and $7 \%$, respectively), but these relapsed cases were salvaged successfully, while relapsed BL and LL showed more dismal outcomes with 5-year OS of $14 \%$ and 17\%, respectively (Fig. 3). Regarding the salvage regimen, patients who received autologous HSCT had a successful outcome with 5-year OS of $77 \%$.

\section{Discussion}

Through this multicenter study, we found that the demographic features and clinical characteristics of pediatric NHL in the East Asia were similar to those of Western countries. However, some distinct aspects were observed.

BL and LL were the most frequent pathologic subtypes followed by DLBCL or ALCL. A previous study analyzed 2,084 patients with pediatric NHL in Western countries and reported that the incidence of $\mathrm{BL}, \mathrm{LL}, \mathrm{ALCL}, \mathrm{DLBCL}$, and primary mediastinal large B-cell lymphoma were $48 \%$, 21\%,
$10 \%, 8 \%$, and $2 \%$, respectively [9]. The pathologic subtype distribution showed different patterns according to the age of patients in western study: BL, LL, and ALCL were more commonly found in children aged under 15 years, while DLBCL, LL, and BL were more frequently found in patients aged 15 years and over [10]. Our study showed similar patterns of pathologic subtype distribution according to patient age as previous studies. In addition, rare pathologic subtypes of lymphoma accounted for $10 \%$ of cases in the 15-19-yearold age group compared to $3 \%$ in the 0-14-year-old age group. PTCL is a pathologic subtype of pediatric NHL, and a BFM study group reported that 38 of 4,083 pediatric patients $(0.9 \%)$ with NHL were diagnosed with PTCL [11]. In our study, PTCL was more frequently found, in 35 (5\%) of 749 patients. Earlier studies showed that in Asia, EpsteinBarr virus (EBV)-associated natural killer (NK)/T-cell lymphomas were more prevalent in adults than they were in Western countries [12,13]. However, there was no significant difference in the distribution of PTCL subtypes in children between the study of the BFM group and our study. In the BFM study, nine of the 38 patients $(24 \%)$ with PTCL were reported to have NK/T-cell lymphomas, compared to 10 of the 35 patients $(29 \%)$ with PTCL in our study. Thus, unlike previous studies on adult NHL, our study found no difference in the rate of NK/ T-cell lymphoma. However, we cannot conclude that there are no ethnic or geographic differences in the biology of PTCL in children. Accordingly, further evaluation related to EBV infection is needed.

The clinical characteristics of pediatric NHL in our study were also similar to those of Western countries. The BFM group study on 2,084 pediatric patients with NHL reported a male to female ratio of 2.7:1 [9], similar to the value in our study (2.4:1). Seventy percent of pediatric patients with NHL were diagnosed with advanced diseases ( $\geq$ stage III) in the 
BFM study, compared to $81 \%$ in our study. While most patients were diagnosed with advanced diseases, BM involvement and CNS involvement were rarely observed. The BFM study observed 5\% CNS involvement and 19\% BM involvement, compared to $8 \%$ and $29 \%$, respectively, in our study. Of note, BM and CNS involvement were more frequently observed in patients with BL and LL than in other subtypes.

Major advances in the treatment of pediatric NHL have been achieved over the last three decades. The current 3- or 5-year EFSs have been reported as approximately 70\% to $90 \%$ in mature B-cell lymphomas, $75 \%$ to $85 \%$ in LLs, and $65 \%$ to $75 \%$ in ALCLs [1-3,14-18]. The development of efficacious treatment strategies for different pathologic subtypes might have resulted in the improved treatment outcomes of pediatric NHL. Our study found that contemporary treatment strategies were successful for treating pediatric patients with NHL in East Asian countries. The treatment outcomes of 749 patients enrolled in our study were comparable with those of Western countries: the 5-year OS and EFS were 89\% and $84 \%$, respectively. Even though treatment protocols differed between institutions, most of the protocols used were based on the backbone of the NHL-BFM or COG protocols. Patients were mostly treated with established protocols stratified to the three main subgroups: LL, mature B-cell lymphoma and ALCL. Therefore, the treatment strategies of each participating institutions were similar to each other. Of note, the treatment outcomes of each institution were not significantly different.

The analysis of prognostic factors in pediatric NHL has been mainly performed for each pathologic subtype in precedent studies. In mature B-cell NHL, advanced stage ( $\geq$ stage III), increased LDH at diagnosis, adolescent age, and CNS involvement have been observed as poor prognostic factors $[19,20]$. A recent BFM study on prognostic factors of mature B-cell NHL showed that presence of mediastinal involvement at the primary site was also a poor prognostic factor, while adolescent age was not associated with prognosis [21]. The COG group also found that disseminated disease, including $\mathrm{BM}$ and CNS, and a high LDH level were poor prognostic factors in mature B-cell NHL [22-24]. In LL, stage at diagnosis was the only identified risk factor, because the study of prognostic factors has been impeded by the rarity of the disease and by difficulties in obtaining appropriate material for immunophenotypic and molecular characterization [3,14]. In ALCL, mediastinal involvement, visceral involvement, or skin lesions were indicated as poor prognostic factors in the study of the European Intergroup for Childhood Non-Hodgkin Lymphoma $[3,25]$. Our study showed similar results: subtype of PTCL, CNS involvement, advanced stage, and high initial LDH level were associated with poor outcomes, whereas age, sex, and BM involvement were not associated with outcomes. A BFM study on pediatric
NHL showed that age and sex had different effects on treatment outcomes and had different distributions according to the pathologic subtype [9]. As such, it may be more reasonable to perform analyses of prognostic factors independently for each subtype.

The outcomes of patients with relapse were dismal. The BFM group reported that $10 \%$ of 324 pediatric patients with LL experienced progression or relapse despite conventional frontline treatment, and the salvage rate was poor with an OS of $14 \%$ [26]. A Korean group study reported an OS of 32\% in refractory or relapsed BL and mature B-ALL [27]. Similar results were noted in our study, showing a 5-year OS of 33\% in relapsed cases. Of note, pathologic subtypes influenced the outcomes. Patients with BL and LL who had refractory or relapsed diseases had a higher rate of treatment failure with conventional salvage therapy than other subtypes: The 5-year OSs of relapsed BL and LL were 14\% and 17\%, respectively, while those of other subtypes were over $50 \%$. These results show that conventional salvage regimens were not efficacious enough in most patients with relapsed BL and LL. Therefore, it is necessary to develop clinical studies to develop new salvage therapy for pediatric patients with relapsed NHLs, especially for BL and LL. Recent multicenter clinical trials of therapies for adult patients with refractory NHL and leukemia showed that blinatumomab and chimeric antigen receptor T-cell therapy could be efficacious salvage therapies [28-30]. This is a possible candidate for salvage therapy in pediatric patients with relapsed and refractory NHL.

As this is a case report form-based retrospective study that recruited a large number of cases from different countries, central review of pathological diagnosis was not possible, and the chemotherapy regimens used in each institution were not uniform. Even with these limitations, our study is noteworthy as the first report to reveal the demographic and clinical features of pediatric patients with NHL in East Asia. The results of this study could be a platform for future collaborative multinational studies in East Asia.

\section{Conflicts of Interest}

Conflict of interest relevant to this article was not reported.

\section{Acknowledgments}

We appreciate TPOG members (especially Chao-Ping, Yang [P.I.]; Meng-Yao, Lu; Hsi-Che, Liu; Kang-hsi, Wu; Chih-Cheng, Hsiao; Jiann-Shiuh, Chen; Shyh-Shin, Chiou; Shih-Chung, Wang; Te-Kau, Chang; Tang-Her, Jaing; Shih-Hsiang, Chen [co-P.I.]; Yung-Li, Yang; Hsiu-Hao, Chang; Ting-Chi, Yeh; Ching-Tien, Peng; Pei-Chin, Lin; Yu-Mei, Liao; Ming-Tsan, Lin; Fang-Liang, Huang; Chao-Neng, Cheng; Jinn-Li, Wang; Wan-Ling, Ho) who contributed his/her patients in this study. 


\section{References}

1. Reiter A, Schrappe M, Parwaresch R, Henze G, Muller-Weihrich S, Sauter S, et al. Non-Hodgkin's lymphomas of childhood and adolescence: results of a treatment stratified for biologic subtypes and stage: a report of the Berlin-FrankfurtMunster Group. J Clin Oncol. 1995;13:359-72.

2. Reiter A, Schrappe M, Tiemann M, Ludwig WD, Yakisan E, Zimmermann $\mathrm{M}$, et al. Improved treatment results in childhood B-cell neoplasms with tailored intensification of therapy: a report of the Berlin-Frankfurt-Munster Group Trial NHLBFM 90. Blood. 1999;94:3294-306.

3. Minard-Colin V, Brugieres L, Reiter A, Cairo MS, Gross TG, Woessmann W, et al. Non-Hodgkin lymphoma in children and adolescents: progress through effective collaboration, current knowledge, and challenges ahead. J Clin Oncol. 2015;33: 2963-74.

4. Lymphoma Study Group, Subspecialty Group of Hematology, the Society of Pediatrics, Chinese Medical Association; Lymphoma Study Group Committee of Pediatrics Chinese AntiCancer Association. A collaborative study of children with lymphoblastic non-Hodgkin's lymphoma in China. Zhonghua Er Ke Za Zhi. 2015;53:931-7.

5. Mitsui T. Recent progress in the treatment of pediatric lymphoma in Japan. Rinsho Ketsueki. 2017;58:2168-77.

6. Hwang IG, Yoo KH, Lee SH, Park YH, Lim TK, Lee SC, et al. Clinicopathologic features and treatment outcomes in malignant lymphoma of pediatric and young adult patients in Korea: comparison of Korean all-ages group and Western younger age group. Clin Lymphoma Myeloma. 2007;7:580-6.

7. Sabattini E, Bacci F, Sagramoso C, Pileri SA. WHO classification of tumours of haematopoietic and lymphoid tissues in 2008: an overview. Pathologica. 2010;102:83-7.

8. Murphy SB. Classification, staging and end results of treatment of childhood non-Hodgkin's lymphomas: dissimilarities from lymphomas in adults. Semin Oncol. 1980;7:332-9.

9. Burkhardt B, Zimmermann M, Oschlies I, Niggli F, Mann G, Parwaresch R, et al. The impact of age and gender on biology, clinical features and treatment outcome of non-Hodgkin lymphoma in childhood and adolescence. Br J Haematol. 2005; 131:39-49.

10. Hochberg J, Waxman IM, Kelly KM, Morris E, Cairo MS. Adolescent non-Hodgkin lymphoma and Hodgkin lymphoma: state of the science. Br J Haematol. 2009;144:24-40.

11. Kontny U, Oschlies I, Woessmann W, Burkhardt B, Lisfeld J, Salzburg J, et al. Non-anaplastic peripheral T-cell lymphoma in children and adolescents: a retrospective analysis of the NHL-BFM study group. Br J Haematol. 2015;168:835-44.

12. Anderson JR, Armitage JO, Weisenburger DD. Epidemiology of the non-Hodgkin's lymphomas: distributions of the major subtypes differ by geographic locations. Non-Hodgkin's Lymphoma Classification Project. Ann Oncol. 1998;9:717-20.

13. Vose J, Armitage J, Weisenburger D; International T-Cell Lymphoma Project. International peripheral T-cell and natural killer/T-cell lymphoma study: pathology findings and clinical outcomes. J Clin Oncol. 2008;26:4124-30.
14. Reiter A, Schrappe M, Ludwig WD, Tiemann M, Parwaresch $\mathrm{R}$, Zimmermann $\mathrm{M}$, et al. Intensive ALL-type therapy without local radiotherapy provides a $90 \%$ event-free survival for children with T-cell lymphoblastic lymphoma: a BFM group report. Blood. 2000;95:416-21.

15. Brugieres L, Deley MC, Pacquement H, Meguerian-Bedoyan Z, Terrier-Lacombe MJ, Robert A, et al. CD30(+) anaplastic large-cell lymphoma in children: analysis of 82 patients enrolled in two consecutive studies of the French Society of Pediatric Oncology. Blood. 1998;92:3591-8.

16. Cairo MS, Krailo MD, Morse M, Hutchinson RJ, Harris RE, Kjeldsberg CR, et al. Long-term follow-up of short intensive multiagent chemotherapy without high-dose methotrexate ('Orange') in children with advanced non-lymphoblastic nonHodgkin's lymphoma: a children's cancer group report. Leukemia. 2002;16:594-600.

17. Williams DM, Hobson R, Imeson J, Gerrard M, McCarthy K, Pinkerton CR, et al. Anaplastic large cell lymphoma in childhood: analysis of 72 patients treated on the United Kingdom Children's Cancer Study Group chemotherapy regimens. Br J Haematol. 2002;117:812-20.

18. Marky I, Bjork O, Forestier E, Jonsson OG, Perkkio M, Schmiegelow $\mathrm{K}$, et al. Intensive chemotherapy without radiotherapy gives more than $85 \%$ event-free survival for nonHodgkin lymphoma without central nervous involvement: a 6-year population-based study from the nordic society of pediatric hematology and oncology. J Pediatr Hematol Oncol. 2004;26:555-60.

19. Cairo MS, Gerrard M, Sposto R, Auperin A, Pinkerton CR, Michon J, et al. Results of a randomized international study of high-risk central nervous system B non-Hodgkin lymphoma and B acute lymphoblastic leukemia in children and adolescents. Blood. 2007;109:2736-43.

20. Patte C, Auperin A, Gerrard M, Michon J, Pinkerton R, Sposto $\mathrm{R}$, et al. Results of the randomized international FAB/LMB96 trial for intermediate risk B-cell non-Hodgkin lymphoma in children and adolescents: it is possible to reduce treatment for the early responding patients. Blood. 2007;109:2773-80.

21. Cairo MS, Sposto R, Gerrard M, Auperin A, Goldman SC, Harrison $\mathrm{L}$, et al. Advanced stage, increased lactate dehydrogenase, and primary site, but not adolescent age ( $\geq 15$ years), are associated with an increased risk of treatment failure in children and adolescents with mature B-cell non-Hodgkin's lymphoma: results of the FAB LMB 96 study. J Clin Oncol. 2012; 30:387-93.

22. Mussolin L, Pillon M, d'Amore ES, Conter V, Piglione M, Lo Nigro L, et al. Minimal disseminated disease in high-risk Burkitt's lymphoma identifies patients with different prognosis. J Clin Oncol. 2011;29:1779-84.

23. Coustan-Smith E, Sandlund JT, Perkins SL, Chen H, Chang M, Abromowitch M, et al. Minimal disseminated disease in childhood T-cell lymphoblastic lymphoma: a report from the children's oncology group. J Clin Oncol. 2009;27:3533-9.

24. Shiramizu B, Goldman S, Kusao I, Agsalda M, Lynch J, Smith 
L, et al. Minimal disease assessment in the treatment of children and adolescents with intermediate-risk (Stage III/IV) B-cell non-Hodgkin lymphoma: a children's oncology group report. Br J Haematol. 2011;153:758-63.

25. Le Deley MC, Reiter A, Williams D, Delsol G, Oschlies I, McCarthy K, et al. Prognostic factors in childhood anaplastic large cell lymphoma: results of a large European intergroup study. Blood. 2008;111:1560-6.

26. Burkhardt B, Reiter A, Landmann E, Lang P, Lassay L, Dickerhoff $\mathrm{R}$, et al. Poor outcome for children and adolescents with progressive disease or relapse of lymphoblastic lymphoma: a report from the berlin-frankfurt-muenster group. J Clin Oncol. 2009;27:3363-9.

27. Kim H, Park ES, Lee SH, Koo HH, Kim HS, Lyu CJ, et al. Clin- ical outcome of relapsed or refractory burkitt lymphoma and mature B-cell lymphoblastic leukemia in children and adolescents. Cancer Res Treat. 2014;46:358-65.

28. Topp MS, Gokbuget N, Stein AS, Zugmaier G, O'Brien S, Bargou $\mathrm{RC}$, et al. Safety and activity of blinatumomab for adult patients with relapsed or refractory B-precursor acute lymphoblastic leukaemia: a multicentre, single-arm, phase 2 study. Lancet Oncol. 2015;16:57-66.

29. Pishko A, Nasta SD. The role of novel immunotherapies in non-Hodgkin lymphoma. Transl Cancer Res. 2017;6:93-103.

30. Batlevi CL, Matsuki E, Brentjens RJ, Younes A. Novel immunotherapies in lymphoid malignancies. Nat Rev Clin Oncol. 2016;13:25-40. 